\title{
Geociências
}

\section{Análise das similaridades das cartas de vulnerabilidade à erosão realizadas por dois operadores zonais de álgebras de mapas}

\section{(Analysis of the similarities of maps of vulnerability to erosion carried out by two zonal operators of map algebra)}

Paulo Sérgio de Rezende Nascimento

Prof. Dr. Eng. Geólogo. Pesquisador CNPq/FAPERN - Laboratório de Geomática Departamento de Geologia, Universidade Federal do Rio Grande do Norte

E-mail: paulo@geologia.ufrn.br

Reinaldo Antônio Petta

Prof. Dr. Geólogo. Pesquisador do Laboratório de Geomática Departamento de Geologia, Universidade Federal do Rio Grande do Norte

E-mail:petta@geologia.ufrn.br

\section{Resumo}

O presente trabalho tem como objetivo comparar as cartas de vulnerabilidade à erosão da sub-bacia do Baixo Piracicaba (SP) realizadas por dois operadores distintos de álgebra de mapas, visando a verificar o nível de semelhança entre os produtos. Esses operadores são denominados de Média Zonal e Maioria Zonal e são comparados, estatisticamente, pela matriz de erros obtida pela tabulação cruzada, índice Kappa e coeficiente de dispersão linear obtido pelo diagrama de dispersão. Pelos resultados obtidos, foi possível verificar que as duas cartas são bastante semelhantes e que fica a critério do analista utilizar um ou outro operador.

Palavras-chave: Média zonal, maioria zonal, álgebra de mapa.

\begin{abstract}
The present work aims to compare the erosionvulnerability maps of the sub-basin of the Low (downstream?) Piracicaba watershed, State of São Paulo, using two distinct operators of map algebra as a way of verifying the level of similarity between the products. These operators are called "Zonal Average" and "Zonal Majority" and are compared statistically using an error matrix, which is obtained by cross tabulation, the Kappa index and the linear dispersion coefficient (obtained from the dispersion diagram). The results show that the two maps are very similar to each other and that the analyst may choose either of the two operators.
\end{abstract}

Keywords: Zonal average, zonal majority, algebra of map. 
Análise das similaridades das cartas de vulnerabilidade à erosão realizadas por dois operadores...

\section{Introdução}

Para se estabelecerem parâmetros e critérios para analisar a capacidade do território em sustentar as atividades antrópicas, um condicionante básico é a carta de vulnerabilidade à erosão. Essa carta é um produto de subsídio ao gerenciamento ambiental, pois indica a inter-relação entre os componentes naturais do meio físico (rocha, relevo, declividade, solo e vegetação) e as formas de ocupação humana frente aos processos erosivos.

Existem vários métodos de classificação do terreno para o mapeamento de áreas vulneráveis aos processos erosivos, com destaque, nesse trabalho, para o sistema de Paisagem, que admite a inter-relação entre os atributos do terreno. Assim, são necessárias tecnologias que permitam o manuseio de dados e informações de diversas naturezas. Essas tecnologias fazem parte do instrumental do geoprocessamento, que utiliza técnicas matemáticas e computacionais para o tratamento das informações (Câmara \& Medeiros, 1996). O programa computacional de geoprocessamento Sistema de Processamento de Informações Georreferenciadas - SPRING (Câmara et al., 1996) permite tratar, de maneira unificada, a grande diversidade de informações cartográficas vetoriais e matriciais (raster) através de operadores algébricos ou de mapas (Erthal et al., 1988).

Esses operadores manipulam um ou mais mapas e suas operações algébricas podem ser pontuais ou zonais. Assim, nesse trabalho, são utilizados dois operadores zonais disponíveis no SPRING para a geração da carta de vulnerablidade à erosão: o Maioria Zonal e o Média Zonal. Esses operadores possuem a mesma finalidade, que é uniformizar, em uma mesma região, os diferentes valores dos atributos utilizados para gerar o produto desejado. Ambos operam sobre um mapa de origem na forma matricial e sobre um conjunto de regiões delimitadas por polígonos na forma matricial ou vetorial, cujo resultado é um mapa matricial (Tomlin, 1990). A diferença entre os operadores é que, no opera- dor Média Zonal, o valor resultante em cada posição geográfica de uma região é igual à média aritmética dos valores da variável geográfica nas mesmas localizações no mapa de origem e, no Maioria Zonal, o valor resultante é igual ao valor de maior freqüência encontrado na mesma região sobre o mapa de origem.

Dessa forma, os produtos gerados por cada operador zonal apresentam diferenças, as quais podem ou não ser significativas. Desta forma, podem surgir dúvidas e indecisões com relação ao qual operador utilizar, haja vista que não há um critério preestabelecido e muito menos nenhuma informação adicional que ajude o analista a optar por um ou por outro operador. Posto isto, o objetivo desse trabalho é avaliar as similaridades entre os produtos gerados (cartas de vulnerabilidade à erosão) por esses dois operadores através de métodos estatísticos como a tabulação cruzada, índice Kappa e diagrama de dispersão. Assim, o trabalho se concentra em determinar a porcentagem e os índices de semelhanças entre as duas cartas de vulnerabilidade à erosão, para que o usuário tenha algum parâmetro de decisão sobre qual operador utilizar.

\section{Material e método}

A área de estudo localiza-se na região central paulista, a nordeste da bacia Sedimentar do Paraná, nesse Estado, delimitada, aproximadamente, pelos paralelos $22^{\circ} 15^{\prime}$ a $22^{\circ} 45^{\prime}$ de latitude Sul e pelos meridianos $47^{\circ} 45^{\prime}$ a $48^{\circ} 30^{\prime}$ de longitude Oeste. Para o desenvolvimento desse trabalho, foram utilizadas as Folhas Topográficas do IBGE, na escala 1:50.000, como segue: Dois Córregos, Brotas, Itirapina, Piracicaba, Capivari, Laras, São Pedro, Barra Bonita e Santa Maria da Serra. As imagens orbitais usadas são as do sensor/satélite TM/Landsat-5, referentes à órbita e ponto 220/73, passagem de 21 de abril de 2002, obtidas no Instituto Nacional de Pesquisas Espaciais (INPE), sem cobertura de nuvens. Para o tratamento digital e interpretação da imagem TM/Landsat, bem como manipular os dados do meio físico utilizaram- se o software SPRING e a linguagem LEGAL (Linguagem Especial para Geoprocessamento Algébrico), ambos desenvolvido pelo INPE.

Os primeiros procedimentos foram a aquisição, compilação e produção dos dados do meio físico-biótico. Assim, os tipos de rochas, relevos e solos foram compilados de Almeida et al. (1981), Ross e Moroz (1997) e Oliveira et al. (1999), respectivamente. As classes de declividade e os tipos de vegetação e uso da terra foram gerados pelos autores desse trabalho.

A próxima etapa foi a integração das informações supracitadas através de processos automáticos de álgebras de mapas. Essa integração é realizada por operadores zonais e pode ser vista como uma extensão da álgebra tradicional. É um conjunto de operadores onde as variáveis manipuladas são regiões geográficas (Berry, 1993) e é utilizado na literatura de Sistema de Informação Geográfica (SIG) e sensoriamento remoto para denotar o conjunto de operadores que manipulam imagens, mapas temáticos e modelos numéricos de terreno.

As operações de zonalidade apresentam restrições espaciais que são definidas por áreas, o que permite materializar num SIG os conceitos de unidade de paisagem preconizados por Tricart (1977) e a área-unidade, que apresenta características individuais próprias.

Essas áreas-unidades formam a base de um sistema de classificação e organização do espaço, onde cada uma delas pode ser relacionada com as demais a partir das características físicobióticas que as individualizam. Foram utilizadas, no presente trabalho, as Unidades Territoriais Básicas (UTB’s), que são unidades de paisagem e que exprimem o conceito geográfico de zonalidade (área-unidade) através de atributos ambientais que permitem diferenciá-las de outras unidades vizinhas, ao mesmo tempo em que possui vínculos dinâmicos que a articulam a complexa rede integrada por outras unidades territoriais (Crepani et al., 1996, Becker \& Egler, 1996). 
Paulo Sérgio de Rezende Nascimento et al.

As operações zonais foram utilizadas para realizar a classificação do grau de estabilidade ou instabilidade à erosão pela atribuição de valores de 1 a 3 , através da análise morfodinâmica da paisagem. Essa análise é feita a partir dos princípios da ecodinâmica (Tricart, 1977), que estabelece uma relação entre processos de morfogênese e pedogênese. Quando predomina a pedogênese, prevalecem os processos formadores de solos e, quando predomina a morfogênese, são os processos formadores do relevo que sobressaem. Dessa análise, podem ser geradas até 21 unidades de paisagem, de acordo com o valor de estabilidade ou vulnerabilidade à erosão que podem ser inseridas em 5 classes de grau de vulnerabilidade (Tabela 1). Essas informações são fornecidas pela geologia, geomorfologia, pedologia, cobertura vegetal e uso do solo, as quais são integradas para que se tenha um retrato do comportamento de cada UTB frente à sua ocupação.

O modelo é aplicado a cada tema individualmente através de operadores de Média Zonal ou Maioria Zonal. Posteriormente recebe um valor final, resultante da média aritmética (operação de atributos) dos valores individuais de cada tema. Após esse procedimento, é realizada a operação de espacialização, que gera o mapa numérico referente à vulnerabilidade média à erosão das UTB's. Só então é feita a operação de transformação de fatiamento, que produz a carta temática de vulnerabilidade à erosão, cujas classes são definidas por valores preestabelecidos e representadas, cartograficamente, por cores ou símbolos distintos.

A comparação entre produtos obtidos por sensoriamento e/ou por geoprocessamento é realizada por métodos estatísticos. A maneira mais comumente utilizada é na forma de uma matriz de erro (Rosenfield \& Fitzpatrick-Lins, 1986, Story \& Congalton, 1986). A diagonal principal indica a porcentagem de similaridade entre os dois produtos e os demais valores da matriz indicam a porcentagem discordante por cada classe.

Tabela 1 - Análise da morfodinâmica da paisagem.

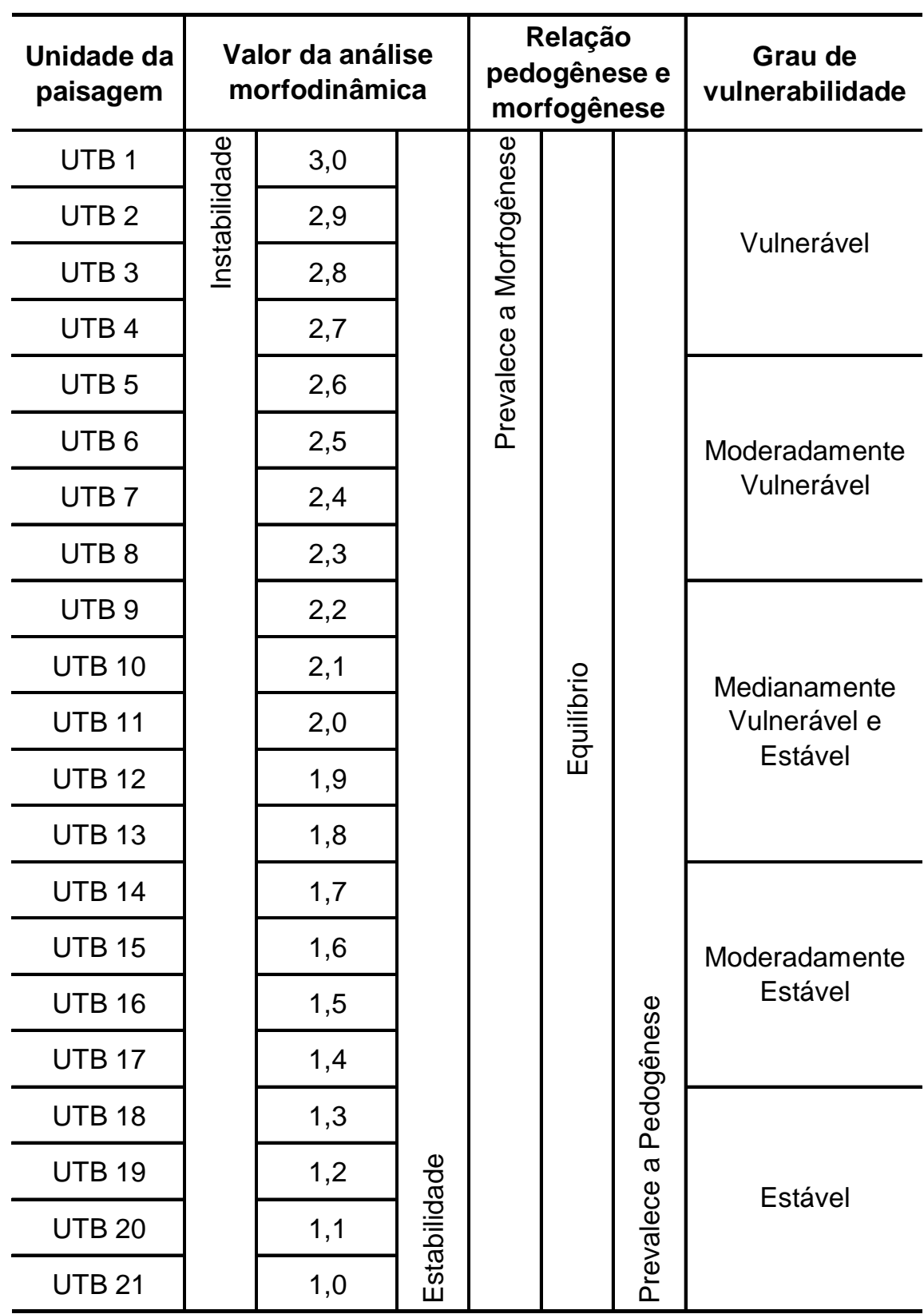

A matriz de erros foi gerada através da técnica de Tabulação Cruzada, que permite calcular e comparar a área das intersecções entre as classes de dois mapas. Posteriormente, essa matriz foi utilizada para aplicação da estatística Kappa. O coeficiente Kappa indica a similaridade existente entre os dois mapas de declividade pelo teste de independência, com nível de confiança de $95 \%$, onde a primeira hipótese (hipótese nula $\mathrm{H}_{0}$ ) é $\mathrm{K}=0$, o que significa que não há concordância, e a segunda hipótese (hipótese alternativa $\mathrm{H}_{1}$ ) é $\mathrm{K}>0$, que indica concordância. Foi adotado o nível de significância igual a 0,05 e o valor de Z, para o correspondente índice Kappa K, foi maior que 1,64 , de forma a rejeitar a primeira hipótese. O valor de Kappa é calculado pelo cruzamento automático das matrizes de erros das duas cartas de vulnerabilidade à erosão pelo algoritmo "Cálculo de Kappa” desenvolvido por Medeiros e implementado por Ponzoni e Almeida (1996), segundo os conceitos preconizados por Congalton et al. (1983). 
Além desses dois métodos, utilizaram-se o diagrama de dispersão para analisar o confronto entre os dois produtos. Esse diagrama é uma representação gráfica para dados bivariados, em que cada par de dados é representado por um ponto de coordenadas, num sistema de eixos coordenados. Ele descreve o relacionamento entre duas variáveis, isto é, entre dois conjuntos de dados ou de duas distribuições. Pelo coeficiente de correlação não se pode afirmar que uma variável influencia a outra, mas é possível estabelecer se existe uma relação e em que intensidade.

Assim, o grau da relação linear entre as variáveis pode ser medido através do coeficiente de correlação. Um coeficiente de correlação é uma medida padronizada do grau da associação entre duas variáveis. É quantificado em uma escala adimensional, que em cada valor absoluto vai de zero à unidade, ou seja, que corresponde a um intervalo de -1 a +1 . O grau de inter-relacionamento entre duas variáveis varia com o módulo do coeficiente, assim quanto maior o módulo do coeficiente, mais intensa é a associação linear entre as duas variáveis. Se o coeficiente de correlação linear for +1 , a associação é positiva perfeita (as duas variáveis crescem juntas), por outro lado, se for -1 , é negativa perfeita (enquanto uma variável cresce a outra diminui) e por fim se for igual a 0 (zero), não há associação entre as duas variáveis.

\section{Resultados e discussão}

Pela simples análise visual entre as Figuras 1 e 2 é possível verificar uma grande semelhança entre elas. A mais marcante é que em ambas não ocorre a classe estável, além disso, as demais classes, apesar de não apresentarem as mesmas porcentagens, apresentam a mesma ordem crescente de representatividade. Assim, a classe moderadamente estável está distribuída na área de estudo em $1,7 \%$ e $2,1 \%$; a classe vulnerável, 3,4\% e 5,8\%: a classe moderadamente estávelvulnerável $30,1 \%$ e $30,2 \%$ e a classe moderadamente vulnerável, 64,8\% e 61,9\%, respectivamente nas cartas de vulnerabilidade à erosão realizadas por média zonal e por maioria zonal.

A Tabela 2 apresenta as matrizes de erros em área do confronto entre as cartas de vulnerabilidade à erosão geradas pelas operações zonais de média e maio- ria zonal. A área total da sub-bacia do Baixo Piracicaba possui 1.463,3 km² e a soma da diagonal principal é $1.304,7$ km², o que indica $89 \%$ de similaridade total entre os dois produtos. A Represa Santa Maria da Serra está inserida na jusante do Rio Piracicaba.

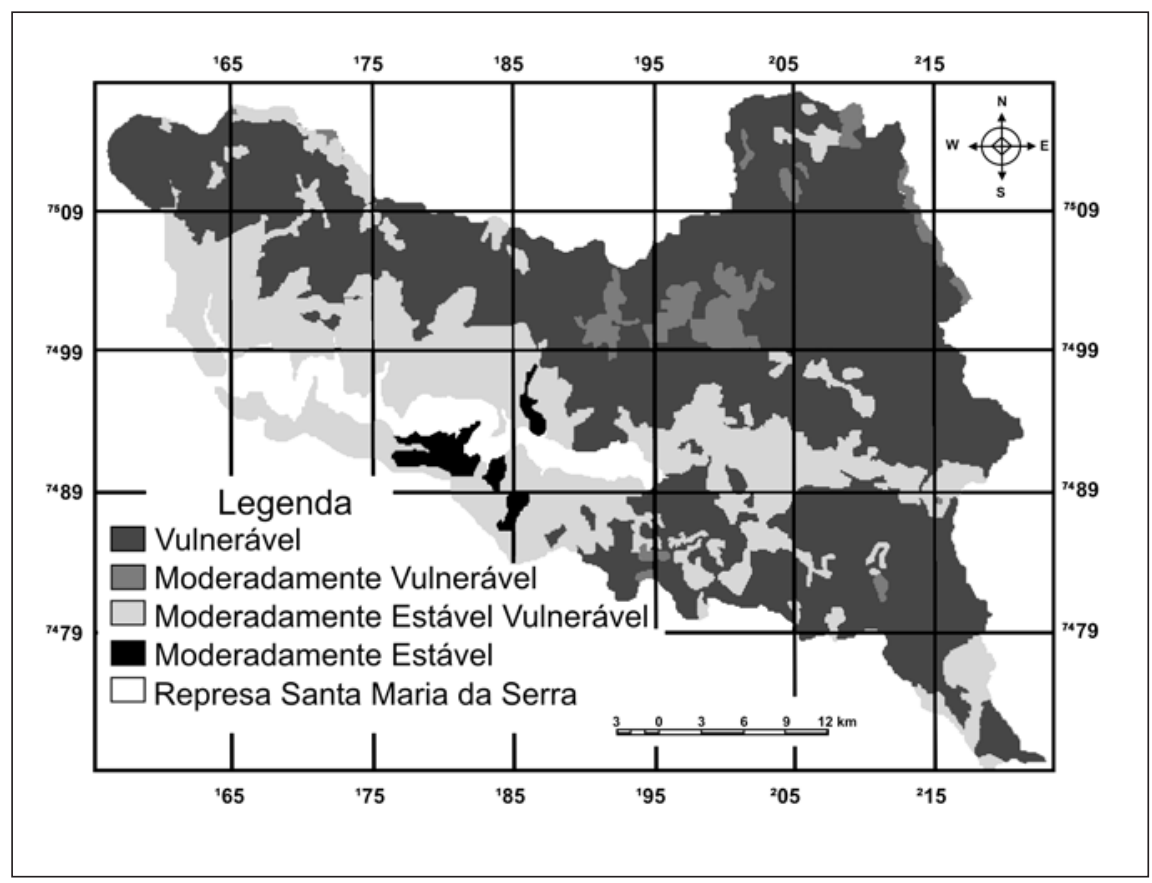

Figura 1 - Carta de Vulnerabilidade à erosão por Média Zonal.

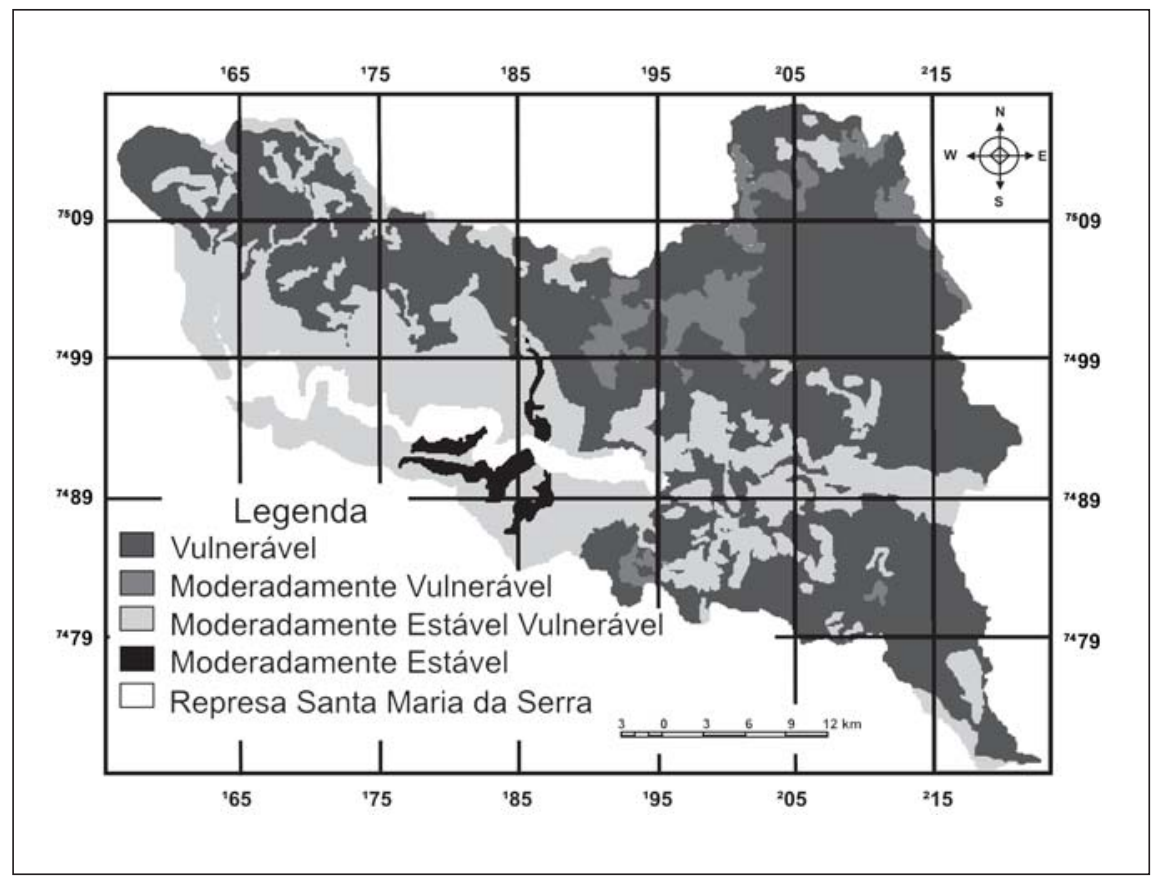

Figura 2 - Carta de Vulnerabilidade à erosão por Maioria Zonal. 
Paulo Sérgio de Rezende Nascimento et al.

Tabela 2 - Matriz de erro em área $\left(\mathrm{km}^{2}\right)$.

\begin{tabular}{c|c|c|c|c|c}
\hline & \multicolumn{6}{|c}{ Maioria Zonal } \\
\hline & $\begin{array}{c}\text { Classes de } \\
\text { Vulnerabilidade }\end{array}$ & $\begin{array}{c}\text { Mod_Estável } \\
\mathbf{( 3 0 , 7 )}\end{array}$ & $\begin{array}{c}\text { Med_Est_Vul } \\
\mathbf{( 4 4 2 , 1 )}\end{array}$ & $\begin{array}{c}\text { Mod_Vulnerável } \\
\mathbf{( 9 0 5 , 8 )}\end{array}$ & $\begin{array}{c}\text { Vulnerável } \\
\mathbf{( 8 4 , 7 )}\end{array}$ \\
\cline { 2 - 6 } & $\begin{array}{c}\text { Mod_Estável } \\
(24,3)\end{array}$ & $\mathbf{2 3 , 7}$ & 0,6 & 0 & 0 \\
$\overline{\frac{\pi}{0}}$ & $\begin{array}{c}\text { Mod_Est_Vulnerável } \\
(439,7)\end{array}$ & 7 & $\mathbf{3 8 5 , 4}$ & 47,3 & 0 \\
\cline { 2 - 6 } & $\begin{array}{c}\text { Mod_Vulnerável } \\
(950,4)\end{array}$ & 0 & 56,1 & $\mathbf{8 5 2 , 6}$ & 41,7 \\
\cline { 2 - 6 } & $\begin{array}{c}\text { Vulnerável } \\
(48,8)\end{array}$ & 0 & 0 & 5,9 & $\mathbf{4 3 , 0}$ \\
\hline
\end{tabular}

O valor de Kappa obtido foi de 0,67 e o de Z foi de 793 e, como o valor de Kappa foi maior que zero e o $\mathrm{Z}$ de maior que 1,64 , entende-se que houve concordância entre os dois produtos, com um nível de confiança de 95\%. Ainda, segundo a avaliação sugerida por Landis e Kock (1977), o valor de Kappa entre 0,6 e 0,8 indica uma similaridade muito boa entre os produtos comparados.

A Figura 3 é o gráfico do diagrama de dispersão entre as cartas de vulnerabilidade à erosão realizada por média zonal e por maioria zonal. O diagrama mostra haver uma associação positiva entre os dois produtos, pois, quando cresce o valor da abscissa, tende a crescer o da ordenada e vice-versa. Essa tendência está quantificada no coeficiente de correlação linear. Esse coeficiente é de 0,92, o que significa que há uma grande correlação entre os dois produtos, ou seja, os produtos são bem similares. No entanto, o fato de ele ser inferior à unidade indica que nem toda a variação de uma distribuição é concomitante com a da outra.

\section{Conclusões}

De acordo com os resultados obtidos pelos testes estatísticos aplicados nos produtos gerados pelos dois tipos de operadores algébricos zonais, eles são bastante similares com 89\% de similaridade obtida pela diagonal principal da matriz de erro, índice Kappa de 0,67 e coeficiente de correlação linear de 0,92.

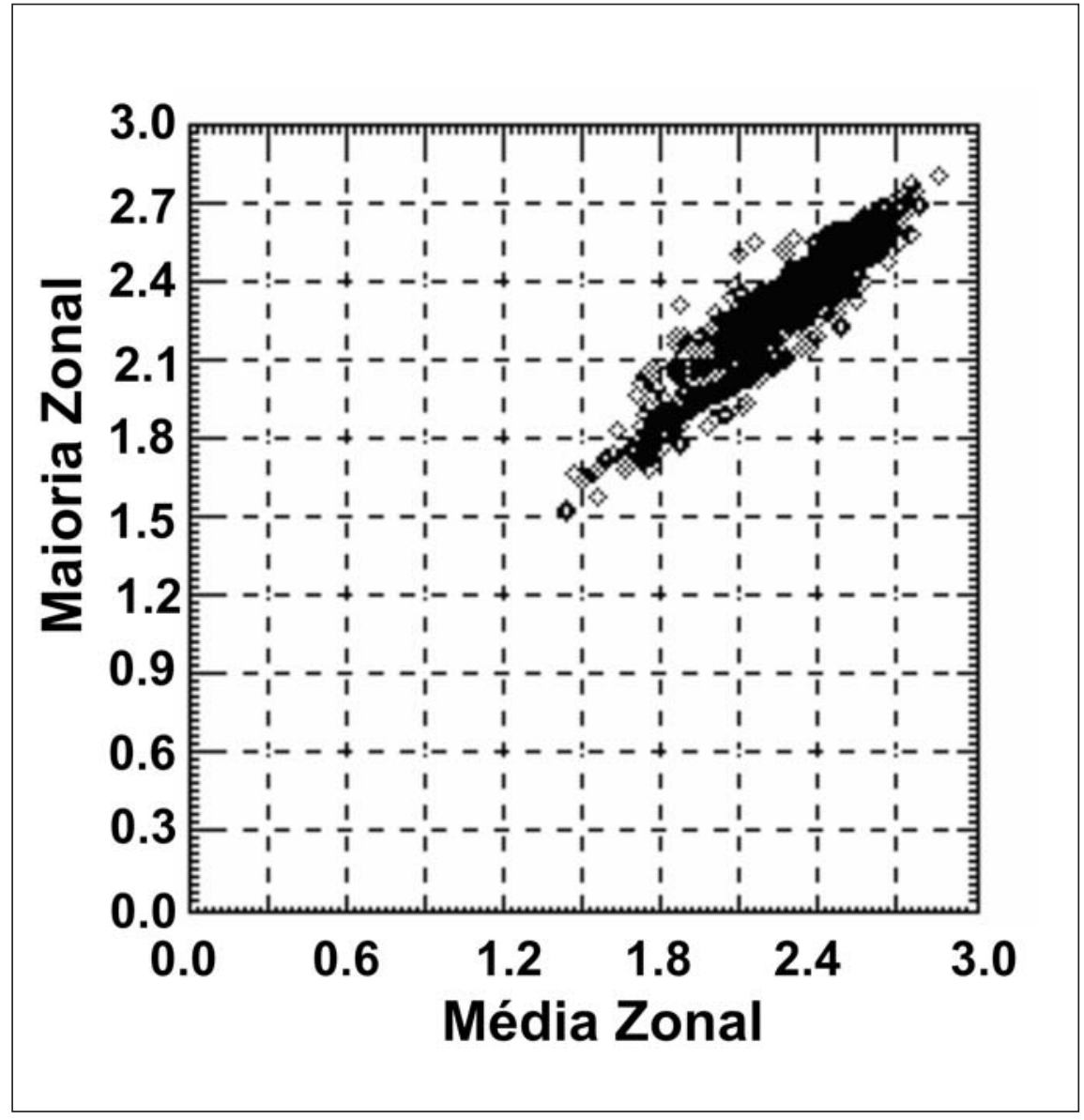

Figura 3 - Diagrama de dispersão entre a Maioria Zonal e Média Zonal.

Assim, apesar de os dois operadores serem conceitual e matematicamente distintos, na prática, os resultados gerados por eles são similares. Deste modo, fica ao critério do analista utilizar um ou outro operador para gerar cartas de vulnerabilidade à erosão através dos procedimentos realizados nesse trabalho. No entanto, não se pode afirmar que esses operadores produzam resultados similares na geração de outros tipos de cartas. 
Análise das similaridades das cartas de vulnerabilidade à erosão realizadas por dois operadores...

\section{Referências bibliográficas}

ALMEIDA, F. F. M. et alii. Mapa geológico do Estado de São Paulo: escala 1:500.000. São Paulo: IPT, 1981. 124p. (Monografia 6).

BECKER, B. K., EGLER, C. A. Detalhamento da metodologia para execução do zoneamento ecológicoeconômico pelos Estados da Amazônia Legal. Brasília: SAE/MMA, 1996. 43p.

BERRY, J. K. Cartographic modeling: the analitical capabilities of gis. In: GOODCHILD, M. et alli. Environmental modelling with GIS. Oxford: Oxford University Press, 1993. 358p. Cap. 5, 58-74p.

CÂMARA, G., MEDEIROS, J. S. Geoprocessamento para projetos ambientais. São José dos Campos: INPE, 1996. 139p.

CÂMARA, G. et alii. SPRING: Integrating remote sensing and GYS by objectoriented data modelling. Computer \& Graphics, v.20, n.3, p.395-403, 1996.

CONGALTON , R. G. et alii. Assessing Landsat classification accuracy using discrete multivariate analysis statical techniques. Photpgrammetric Engineering and Remote Sensing, v.49, n.12, p.1671-1678, 1983.

CREPANI, E. et alii. Sensoriamento remoto aplicado ao zoneamento ecológicoeconômico. São José dos Campos: INPE, 1996. 18p.

ERTHAL, G. et alii. Modelo de dados georelacional: uma visão conceitual de um sistema geográfico de informações. In: SIMP. BRAS. COMP. GRÁF. PROC. IMAG., 1. 1988. Petrópolis. Anais... Petrópolis. p. 1-10. 1988.

LANDIS, J., KOCK, G. G. The measurements of observer agreement for categorical data. Biometrics, v.33, n.3, p.159-174, 1977.

MEDEIROS, J. S. Desenvolvimento metodológico para a deteção de alterações da cobertura vegetal através da análise digital de dados MSS/ Landsat. São José dos Campos: Instituto Nacional de Pesquisas Espaciais, 1987. 81f. (Dissertação de Mestrado em Sensoriamento Remoto).

OLIVEIRA, J. B., CAMARGO, M. N., ROSSI, M., CALDERADO FILHO, B. Mapa pedológico do Estado de São Paulo: Escala 1:500.000. Campinas:IA, 1999. 63p.
PONZONI, F. J., ALMEIDA,E. S. A estimativa do parâmetro kappa (K) da análise multivariada discreta no contexto de um SIG. In: SIMP. BRAS. DE SENS. REM., 8. 1996. Salvador. Anais... São José dos Campos, p.14-19, 1996.

ROSENFIELD, G. H., FITZPATRICKLINS, K. A coefficient of agreement as a measure of thematic classifications accuracy. Photogrammetric Engineering and Remote Sensing. New York: McGraw-Hill, v. 52, n.2, p.223-227, 1986.

STORY, M., CONGALTON, R. G. Accuracy assessment: a users perspective. Photogrammetric Engineering and Remote Sensing. New York: McGrawHill, v. 52. n.3 p.397-399, 1986.

ROSS, J. L. S., MOROZ, I. C. Mapa geomorfológico do Estado de São Paulo: escala 1:500.000. São Paulo: USP;IPT;FAPESP, 1997. 64p.

TOMLIN, D. Geographic information systems and cartographic modeling. New York:Prentice Hall, 1990. 249p.

TRICART, J. Ecodinâmica. Rio de Janeiro: IBGE, 1977. 91p.

Artigo recebido em 24/08/2007 e aprovado em 25/02/2008.

\title{
$* * * * * *$
}

\section{REM - Revista Escola de Minas 72 anos divulgando CIÊNCIA.} $* * * * * *$

www.rem.com.br

\author{
$* * * * * *$
}

